\title{
Searches for Double Parton Scattering at the LHC
}

\section{Miroslav MYSKA*}

Institute of Physics, Academy of Sciences of the Czech Republic, Na Slovance 2, CZ - 18221, Prague, Czech Republic

E-mail: myskamdfzu.cz

Searches for the kinematic selection criteria for estimation of multiple parton interaction fraction in pp collisions at $\sqrt{s}=14 \mathrm{TeV}$ are presented using the eikonalization of the cross section implemented in the Herwig++ Monte Carlo event generator. $\mathrm{W}^{+}$boson pair production is studied in the muon decay channel. Four types of the main background processes are discussed and analyzed. These include $W^{+} W^{+} j j, W^{+} Z, Z Z$, and $t \bar{t}$ productions of the positively charged muon pairs. The double parton scattering contribution to the requested di-muon final state is found to be around 25 per cent with the production cross section of $0.94 \mathrm{fb}$.

XXIst International Europhysics Conference on High Energy Physics

21-27 July 2011

Grenoble, Rhône-Alpes France

${ }^{*}$ Speaker. 


\section{Introduction}

The topic of the Multiple Parton Scattering (MPS) is discussed within the hadron collider physics for many years. The main motivation for their consideration is to explain the high activity in detectors in comparison to the standard picture of Single Parton Scattering (SPS), especially for the low transverse momentum multi-jet production. The parton model allows us to consider several independent soft or semi-hard parton-parton interactions within the same hadron-hadron collision and so model the production of many particles in the final state. All the three general-purpose Monte Carlo event generators Herwig++ [四], Pythia8 []], and Sherpa [3], have implemented their mechanisms in order to model MPS.

\section{2. $\mu^{+} \mu^{+}$Production and SPS Background Estimation}

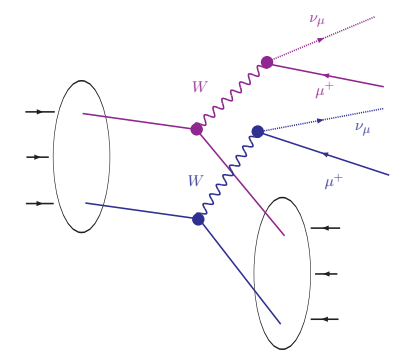

Figure 1: Double parton scattering in protonproton collision. A pair of $W^{+}$bosons is produced via two independent parton annihilations.

The vector boson pair production offers a very straightforward test of the current stage of the MPS models. Despite the multijet production, the double Drell-Yan process combines only limited variations of quark-antiquark flavor pairs and can probe the universality of the common normalization factor often called effective cross section. The simultaneous production of two positively charged $W$ bosons (see Fig.W) was chosen to be probed at the Monte Carlo level as the prediction for the following measurement at the LHC experiments. The double parton scattering signal was generated using the Herwig++ program which is fully capable to generate this type of proton-proton events within its underlying event model based on the eikonalization of the cross section. In order to satisfy the inclusivity of this DPS process in the number of parton subinteractions in one event, Herwig++ generates several $Q C D 2 \rightarrow 2$ sub-processes in addition to two Drell-Yan annihilations according to the pre-sampled distribution of the Poissonian shape [四].

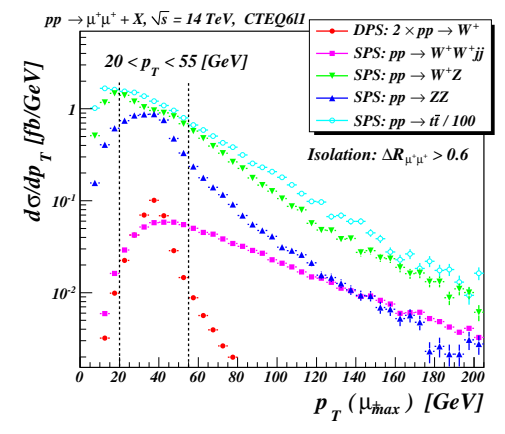

(a)

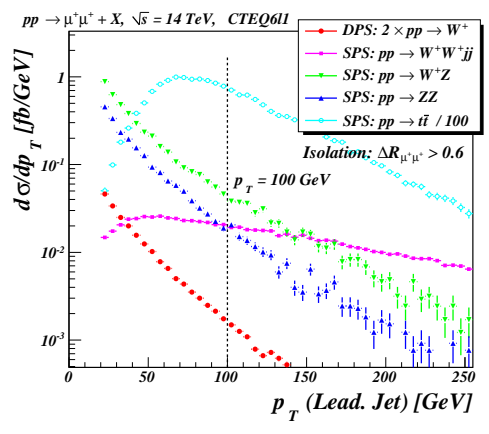

(b)

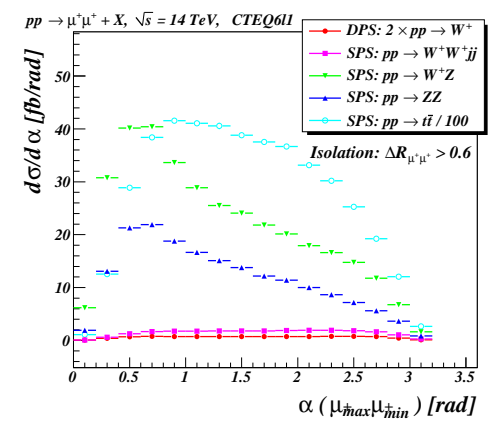

(c)

Figure 2: Differential cross section as a function of (a) the transverse momentum of the hardest positively charged muon, (b) of the transverse momentum of the leading jet, (c) of the relative angle between the measured muons. 
Four types of physics background is studied in order to establish the kinematical region, where the double parton scattering production mechanism has a maximal significance against other processes producing two same-sign muons in the final state. The vector boson pair productions for three charge combinations: $W^{+} W^{+}, W^{+} Z$, and ZZ, are prepared using the MadGraph/MadEvent generator [G], where $Z$ stands for both $\gamma$ and $Z$. Heavy flavor quark pair production is generally the dominant source of the searched di-muons because of the huge cross section, that leaves lot of space for the muon radiation from short-living hadrons together with the heavy quark decay to the hard muon. The $t \bar{t}$ production is studied using the Herwig++ generator.

An example of the kinematical comparison among the individual contributions to the di-muon final state is shown in Fig.[. The $p_{T}$ spectrum of the two hardest muons $\left(\mu_{\max }^{+}\right.$and $\left.\mu_{\min }^{+}\right)$in the event is the strongest selection tool together with the jet analysis. The final result is also significantly dependent on the minimal $p_{T}$ of the negatively charged muon $\left(\mu_{m a x}^{-}\right)$that can be measured within the given detector acceptance. The final selection criteria are summarized in Table $\mathbb{l}$. Jets are clustered using the anti $-k_{t}$ algorithm [ [6] implemented in the FastJet package [四] with $R=0.4$.

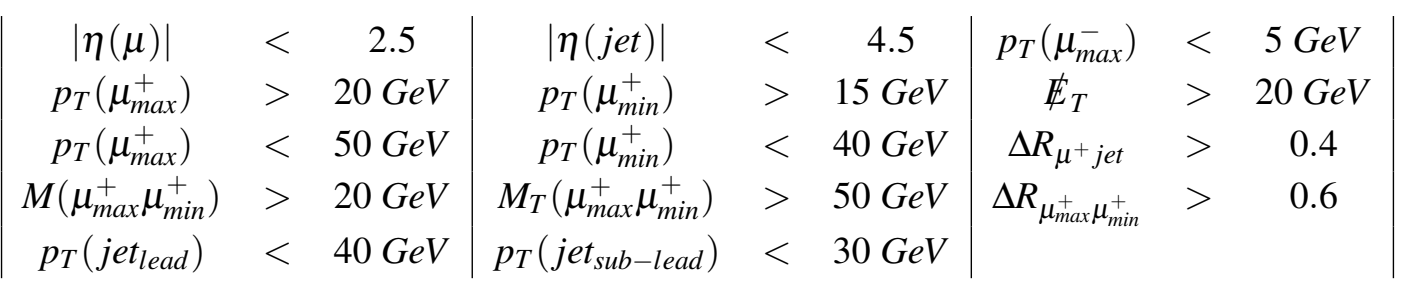

Table 1: Summary of the final selection criteria applied on Monte Carlo data.

\section{Summary and Conclusion}

Several mechanisms were studied in order to find the kinematical region for the statistically reasonable measurement of the double parton scattering. The final selection described in Table $\square$ is considered as the main set of the selection criteria, where signal-background ratio reaches 0.35 . The result will also strongly depend on the detector performance and on the trigger efficiencies. Considering that the signal cross section in $\mathrm{LO}$ is $0.94 \mathrm{fb}$, the integrated luminosity is required to be very large, of $\mathscr{O}\left(100 \mathrm{fb}^{-1}\right)$. However, the LHC could provide enough of the necessary statistics within a few years of full energy operation. Measurement of this process may bring a significant insight into the hadron structure, especially for the transverse parton distributions, and very valuable feedback for Monte Carlo generators.

\section{References}

[1] M. Bähr at al., Eur. Phys. J. C58 (2008) 639

[2] T. Sjöstrand, S. Mrenna and P. Skands, JHEP05 (2006) 026

[3] T. Gleisberg at al., JHEP02 (2009) 007

[4] I. Borozan and M. H. Seymour, JHEP09 (2002) 015

[5] J. Alwall at al., JHEP09 (2007) 028

[6] M. Cacciari and G. P. Salam, JHEP04 (2008) 063

[7] M. Cacciari, G. P. Salam and G. Soyez, Phys. Lett. B641 (2006) 57 